\title{
Asuntos teóricos y metodológicos de la cultura organizacional* $^{*}$
}

\author{
Theoretical and methodological matters of organizational \\ culture \\ Recibido: 12 de febrero de 2009 - Revisado: 27 de febrero de 2009 - Aceptado: 03 de julio de 2009 \\ Claudia Eugenia Toca Torres $^{* *}$ - Jesús Carrillo Rodríguez ${ }^{* * *}$
}

\section{Resumen}

Este documento recoge algunos elementos constitutivos de la Cultura Organizacional (CO), reflexionando sobre métodos relevantes para su estudio. Destaca que la importancia de caracterizarla y medirla radica en su impacto tanto en los resultados directos y la eficiencia de una organización, como en la salud, el entusiasmo, el compromiso y la flexibilidad de sus miembros. Advierte, finalmente, que la responsabilidad no termina con su medición, sino que exige complementariamente la dirección y el moldeamiento de acuerdo con las circunstancias del entorno.

\section{Palabras clave}

Cultura Organizacional, eficiencia organizacional, acción colectiva

\begin{abstract}
This paper is concerned with Organizational Culture (OC), its components and the main techniques to study it. It states that measuring and establishing its impacts related with results, efficiency, healthy and welfare of employees are important issues for any manager - public or private. But measure culture is not enough, we need to be engage with both manage and shape it according to the organizational context.
\end{abstract}

\section{Key words}

Organizational Culture, organizational efficiency, class action

\footnotetext{
* Artículo resultado de Investigación.

** Doctora en Ciencias Políticas y Sociales, Maestra en Administración y Especialista en Administración de la Universidad Nacional Autónoma de México, UNAM. Profesor Asociado de Carrera de la Facultad de Administración de la Universidad del Rosario.

Correo electrónico:

claudia.toca@urosario.edu.co.

*** Doctor en Ciencias Políticas y Sociales de la Universidad Nacional Autónoma de México, UNAM, Maestro en Gobierno y Asuntos Públicos de la Facultad Latinoamericana de Ciencias Sociales FLACSO Sede México, Docente Facultad de Ciencia Política de la Universidad Javeriana.

Correo electrónico:

jesus.carrillo@javeriana.edu.co.
} 


\section{Introducción}

Los teóricos sociales han estudiado, de tiempo atrás, la acción colectiva y sus problemas, intentando determinar las formas adecuadas para organizar la actividad humana y así alcanzar determinadas metas sociales. De este legado conviene reseñar el estrecho vínculo y la indisoluble relación entre organización y acción colectiva, pues cada vez que se persiguen propósitos comunes, estos demandarán de un conjunto de personas que colaboren entre sí para alcanzar aquello que individualmente resultaría imposible. La organización, en consecuencia, constituye una premisa de todo emprendimiento colectivo.

Son muchas las acepciones encontradas cuando se estudia la organización. Efectivamente, la organización puede considerarse indistintamente como un sistema, una jerarquía o un mecanismo de propósitos; o bien como centros de elección y decisión para tratar problemas comunes. Como institución, la organización explica la interacción humana y el desempeño en sus dimensiones social, política o económica ${ }^{1}$. Así, uno de los más destacados institucionalistas, Douglas North (1994), destaca el papel de las organizaciones en el cambio institucional e invoca la necesidad de estudiar las estructuras de gobernación y las capacidades para explicar el éxito de las sociedades a lo largo del tiempo.

Los países latinoamericanos han entendido esta necesidad y han emprendido sendas reformas institucionales en las últimas décadas, sin embargo un atributo permanentemente ignorado en estos esfuerzos reformistas es la Cultura Organizacional (CO). Se aprecia, por ejemplo, que las iniciativas para alcanzar modelos posburocráticos en el sector público han sido insensibles a las consideraciones culturales de la organización, y en consecuencia, el desempeño nunca reflejará avances sustanciales y sostenidos. Es por ello que la "transformación cultural" es exigida como un medio para introducir mejoras en la calidad y el desempeño gubernamental, ubicando al conjunto de referentes culturales como determinante del éxito en la provisión de bienes y servicios públicos. Las organizaciones oficiales presentan las mismas características básicas de otras, sin embargo revelan algunas especificidades (apego a las reglas y rutinas, supervaloración de la jerarquía, paternalismo en las relaciones y adhesión al poder) que resultan importantes para la definición de elementos y procesos internos (Pires et al. 2006. pág. 12).

Originados en la antropología social, hoy los estudios de la $\mathrm{CO}$ resultan de interés para otras disciplinas como la psicología, la sociología, la administración y la ciencia política. Actualmente, constituye un campo de investigación que genera muchos debates por esta confluencia disciplinaria y cuyas diferencias se centran fundamentalmente en los aspectos ontológicos, epistemológicos, metodológicos y de perspectiva, generalmente utilizados para enriquecer la discusión de tan promisoria línea de investigación. La cultura ha sido tratada como variable desde la perspectiva racionalista y funcionalista, el interés se ha centrado en determinar la función que cumple en la organización, y como metáfora desde la óptica simbólica con el propósito de establecer el significado de la organización para sus miembros (Alvesson 1993; Schultz 1995 en Rowlinson et al. 1999. pág. 1).

En esta línea, este documento aborda uno de los asuntos más difíciles de ponderar en las organizaciones: su cultura. En un primer momento resalta algunas diferencias entre las distintas propuestas conceptuales de $\mathrm{CO}$, posteriormente reflexiona sobre sus dimensiones, componentes y tipologías, y por último, centra su atención en las técnicas disponibles para su medición. Presenta de manera sucinta los asuntos básicos en el análisis cultural de las organizaciones, sirviendo como punto de partida para aquellos interesados en mejorar la acción colectiva o el desempeño social, definiendo la cultura organizacional como una variable independiente y multidimensional. 


\section{Significadodelacultura organizacional}

Organizaciones formales e informales se rigen por valores, normas, convenciones y tradiciones, que cambian con el tiempo pero condicionan la actuación de los individuos. En la literatura, las normas en las organizaciones son conocidas como cultura (Fitzgerald. 2002. pág. 103).

La cultura en términos generales se concibe como un sistema de significados que genera algún tipo de identidad compartida (Geertz, 1989), una especie de código que orienta las prácticas sociales de personas pertenecientes a varios grupos y categorías sociales dentro de una sociedad (Vaitsman, 2000. pág. 848).

A partir de la génesis del concepto de cultura se establecen cuatro categorías fundamentales: 1) Cognitiva, en la que la cultura se vuelve inteligible como un estado mental general, soportando la idea de la perfección, del logro individual o de la emancipación. 2) Colectiva, que invoca un estado de desarrollo intelectual o moral en la sociedad, vinculando la cultura con la idea de la civilización. 3) Concreta y descriptiva, ve la cultura como un cuerpo de destrezas y trabajos intelectuales dentro de cualquier sociedad, como el campo de simbolismos producidos y sedimentados de una sociedad. 4) Social, relaciona la cultura con la forma integral de vida, en un sentido plural y potencialmente democrático (Jenks, 1993. págs. 11-12).

Bajo ninguna circunstancia, la concentración geográfica de las organizaciones garantiza la homogeneidad en sus culturas internas. Por el contrario, en ocasiones las diferencias son tan marcadas como las que podrían existir entre instituciones de distintas localidades, regiones o países. Si a esto se agregan las diferencias propias de los sectores en los cuales se encuentran inmersas, el resultado final será una diversidad en la Cultura Organizacional, CO. A este respecto, autores como Herbert (2000: 148) concluyen que la principal influencia de la $\mathrm{CO}$ es la idiosincrasia nacional o regional del país donde opera, en tanto la industria o sector que enmarca sus actividades ayuda a moldear sus valores culturales.

El desarrollo de la cultura es lo que distingue la sociedad humana de las sociedades de otras especies, la diferencia radica en las formas de actuar, pensar y sentir transmitidas de una generación a otra a través del aprendizaje (Albrow, 1999. pág. 6). A su vez, la cultura en una organización está relacionada con la forma como los miembros comparten un marco de referencia común y enfrentan los cambios. Frente a culturas dispersas, la gente tendrá diferentes percepciones e interpretaciones de los cambios organizacionales $\mathrm{y}$, por consiguiente, esquemas diversos sobre el cambio (Lau and Woodman, 1995 en Lau et al. 2002. pág. 539).

Derivado de la cantidad de disciplinas que han estudiado la cultura para explicar fenómenos concretos, un sinnúmero de definiciones complejas y divergentes aparecen en la bibliografía especializada. Al interior de las Ciencias Sociales por ejemplo -especialmente en estudios de antropología social, de sociología, de psicología social y organizacional-, existe poco consenso en torno al significado del concepto, dadas las diferentes representaciones que intervienen en la formación del mismo. Los estudios organizacionales y gerenciales, por ejemplo, han atribuido de manera entusiasta el concepto de cultura a fuentes antropológicas y sociológicas. Desde el referente de la antropología social la cultura se considera como un conjunto compartido de valores sobre asuntos de relevancia societal, tales como la vida, la muerte, las relaciones de consanguinidad, la religión y la etnia de sus miembros; desde la perspectiva sociológica, como el agregado de relaciones que sirven y corresponden a las necesidades de la estructura social en los agrupamientos de trabajo ${ }^{2}$.

La cultura organizacional nace, se materializa y se institucionaliza en conductas 
y acciones. Concretamente, ha sido definida como el conjunto de normas o mandatos aprendidos por los nuevos miembros, gracias a la conciencia que toman de lo que significa una conducta aceptable o una inaceptable. Esta consideración descarta la imposición mediante la formalización de reglas escritas, por 1o que resulta difícil encontrarla consignada en algún tipo de documento - manual, código o prontuario-, en su lugar, es transmitida de manera casual. Es así como muchas organizaciones del mundo han demostrado que el éxito se ha derivado de las reglas no escritas y los entendimientos compartidos alrededor del balance entre autonomía y control, sin centrarse en procedimientos formalizados o jerarquías rígidas. Central para los propósitos de este escrito, a continuación se profundizará en dicho concepto.

Sin lugar a dudas, el autor más destacado es Edgar H. Schein, quien define la CO como un modelo de supuestos básicos compartidos -inventados, descubiertos o desarrollados por un grupo determinado, al ir aprendiendo a enfrentar sus problemas de adaptación externa e integración interna- que hayan ejercido la suficiente influencia como para ser considerados válidos $\mathrm{y}$, en consecuencia, ser enseñados a los nuevos miembros como el modo correcto de percibir, pensar y sentir esos problemas (1988: 25). Por su parte, y de modo sencillo, George Herbert la caracteriza como el pegamento que mantiene unida la organización y que incorpora valores, normas de conducta, políticas y procedimientos (2000: 148).

La cultura fue pensada en términos de un entendimiento social compartido, producto de supuestos y visiones comunes entre los miembros de una organización. Desarrollada en una colectividad a través de experiencias compartidas, por lo regular, durante largos periodos de tiempo permitiéndoles a sus miembros coordinar actividades sin tener que lograr acuerdos explícitos en cada instancia (Wilkins and Ouchi, 1983; Schall, 1983;
Rousseau, 1990; Schein, 1983, en Weber et al. 2003. pág. 402).

Paralelamente, autores como Kennedy y Deal la consideran un modelo integrado de conducta humana que incluye, además del habla y la acción, ciertos artefactos, destacando la capacidad del hombre para obtener y transmitir conocimiento y para heredarlo a otras generaciones (1982: 5). Finalmente, para Stanley Truskie constituye un conjunto de valores y prácticas compartidos a través de todos los grupos, una fuerza poderosa para moldear la conducta del empleado y factor significativo para promover e inhibir el desempeño organizacional (1999: 5).

A partir de los anteriores autores y para efectos de una propuesta conceptual, es posible definir la CO como el conjunto de instituciones informales compartidas que gobiernan $y$ legitiman la conducta de una colectividad organizacional. En esta concepción, el carácter informal pone distancia a los mecanismos manifiestos $\mathrm{u}$ oficiales como pueden ser los reglamentos, las políticas, los manuales o los mandatos. Son los estudios sociológicos los que se han concentrado en las normas informales, definidas como reglas de un grupo o comunidad que pueden o no ser explícitamente establecidas y que dependen de mecanismos de monitoreo tales como la aprobación o desaprobación social. Las normas que gobiernan las relaciones interpersonales limitan y permiten conductas mediante la definición de estructuras de incentivos (Brinton et al. 2001. pág. 19). Los analistas organizacionales por su parte, distinguen entre la arquitectura formal y las características culturales informales. La primera referida a las especificaciones oficiales de una organización y sus formas de gobernación. La cultura, por su parte, establece de manera informal cómo deber ser realizado el trabajo, cómo interactúan los miembros, cómo se toman las decisiones, cuáles unidades difieren de otras, etc. La noción de cultura incluye el conocimiento tácito y el conocimiento generado fuera de la organización y las normas 
que codifican los entendimientos y prácticas informales de interacción, autoridad y demás (Hannan et al. 2003. pág. 401).

La cultura organizacional proporciona las reglas de gobierno de los asuntos cognitivos y afectivos en una organización, así como los medios para ser moldeados y expresados (Kunda, 1992 en Alvesson, 2002. pág. 3). Las reglas de actuación de origen cultural-formales e informales-adquieren racionalidad a través de los procesos de interacción entre los distintos agentes, hasta que alcanzan un nivel de consolidación o institucionalización que determina la configuración de sus percepciones, sus intereses y el tipo de interrelaciones que se producen entre ellos. La percepción del mundo que tienen los individuos está determinada por la cultura institucional en la que se desempeñan. Individuos y organizaciones sólo pueden actuar a partir de los valores y del conocimiento que les proporciona su marco institucional (Zurbriggen, 2006. pág. 71).

Ha sido aceptada la idea de que la cultura está constituida por elementos y procesos reflejados en las acciones de la organización y es transmitida y asimilada por sus miembros a través del diario compartir (Woida et al. 2006. pág. 48). La CO puede ser considerada como un activo o como una obligación, según las circunstancias. Cuando toma la forma de activo genera altos niveles de cooperación, dada la existencia de unas creencias y valores compartidos. Como obligación, demanda obediencia y subordinación, impidiendo una adecuada alineación de creencias y valores organizacionales con los personales (Frew, 1996: 2).

\section{Dimensiones y componentes de la cultura organizacional}

A pesar de los avances registrados en su estudio, el tema de la cultura organizacional resulta complejo y en ocasiones hasta difuso. Se encuentran propuestas, críticas e intentos realizados por los estudiosos para esclarecer y definir el término, como también en las cuestiones ontológicas (del ser) y metodológicas del estudio de las organizaciones y su cultura ${ }^{3}$. Interesa registrar tales asuntos, especialmente en sus dimensiones y componentes.

Debido a que la cultura involucra fenómenos sociales que ayudan a definir el carácter y las normas de la organización, el rango de componentes es amplio y variado. Para facilitar la labor de identificación, Schein (1985) y Lundberg (1990) han establecido un marco de tres capas o dimensiones básicas que encuadran sus distintos elementos. En consonancia $-\mathrm{y}$ a partir de la conceptualización de Schein-, otros estudiosos han asimilado las capas de la cultura a las de una cebolla (ver figura 1), donde la capa externa comprende aspectos observables, mientras que las internas encarnan lo invisible o idealizado (National Research Council Staff, 1997: 68).

\subsection{Dimensión esencial}

Aquella definida como el conjunto de premisas o preceptos que impactan vigorosamente la gran mayoría de pensamientos y acciones organizacionales. Es considerada la capa más profunda y está constituida por cuatro componentes: los valores, los supuestos, las ideologías y el conocimiento (Lundberg, 2000: 701).

Figura 1

Dimensiones de la cultura organizacional

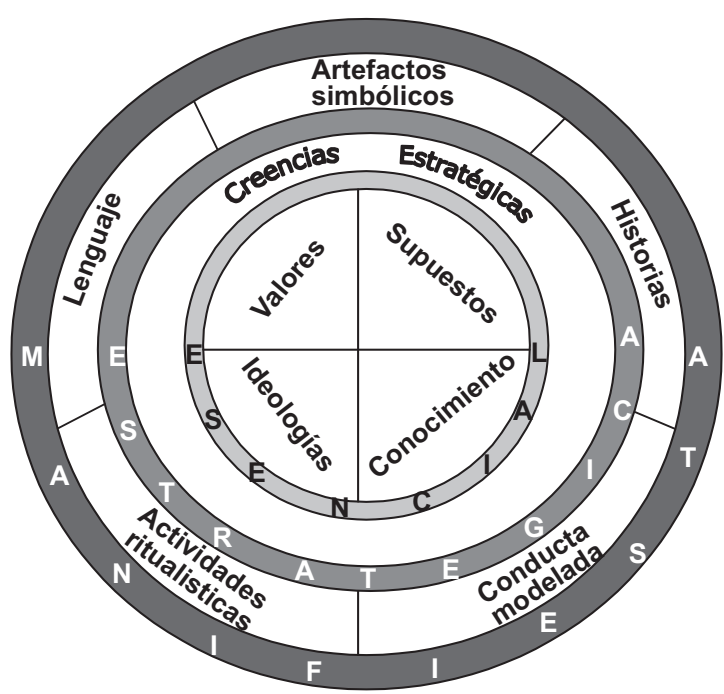

Fuente: Elaboración propia 
Los valores enmarcan las ideas abstractas compartidas por los miembros de una colectividad, que proporcionan un sentido de dirección común sobre lo que es deseable y correcto, definen un carácter y una actitud distintiva. Los supuestos representan las premisas, percepciones, pensamientos y sentimientos compartidos, en los que se fundamentan las visiones del mundo, refieren de igual modo aspectos básicos como la naturaleza de las relaciones humanas, de la verdad, del tiempo y de la actividad humana. Las ideologías se consideran como un sistema coherente de credos compartidos e interrelacionados que dan explicaciones generales convincentes -en algunas ocasiones míticas- sobre la realidad social, exhortandoalaacción colectivayalacomunicación e interpretación de los acontecimientos de cada día. Por último, se establece que el conocimiento es la manifestación perceptual de valores y es referido a las representaciones, juicios, explicaciones y entendimiento, establecidos como racionales para la acción y que evidencian la habilidad de los miembros para interpretar hechos con base en el acceso a información y a marcos cognitivos.

\subsection{Dimensión estratégica}

Las creencias estratégicas son el único componente de este nivel intermedio y no tienen quevercon planesa largo plazo o pronunciamientos de los voceros organizacionales, sino más bien con convicciones y certezas de sus líderes. Esto en virtud a que buscan emplear todos los elementos del poder para lograr los objetivos. Las creencias culturales son las ideas y pensamientos comunes a diversos individuos que gobiernan su interacción, difieren del conocimiento ya que no son descubiertas empíricamente ni analíticamente probadas. Por lo general son conocidas mediante procesos de socialización por los que la cultura es unificada, mantenida y comunicada (Brinton et al. 2001. pág. 78). Existen cuatro tipos básicos (Lundberg, 2000: 701): Creencias sobre la visión, referidas a lo que la organización puede llegar a ser y lo que nunca debería intentar; Creencias sobre las expectativas del mercado de capital, convicciones sobre lo que se necesita para mantener a acreedores e inversionistas satisfechos; Creencias sobre la competencia en el mercado, entendimiento de cómo y por qué se puede triunfar en el entorno, industria o sector; y Creencias sobre la dirección interna, prácticas operativas adecuadas que sustentan los tres tipos anteriores. La dimensión estratégica refleja, por un lado, cierta esencia cultural y por otro, acondiciona de manera activa los elementos manifiestos, es decir, los más superficiales o visibles.

\subsection{Dimensión manifiesta}

Los elementos de esta dimensión externa son relativamente visibles y llevan a que los miembros identifiquen de forma similar los problemas y experimenten de manera semejante eventos, actividades y situaciones organizacionales, dentro de los límites considerados como aceptables y hacia propósitos comunes. Este nivel enmarca el "know how", es decir todo tipo de prácticas institucionales, como los artefactos simbólicos, el lenguaje, las historias, las actividades ritualistas y la conducta modelada (Lundberg, 2000: 705). Los símbolos y manifestaciones producidas por un grupo se reconocen como artefactos, considerando entre ellos los espacios físicos-instalaciones, mobiliario- -y la capacidad tecnológica -mecanización, sistematización, saber hacer. (Schein, 1988: 31). El lenguaje en la organización incluye el vocabulario único o jerga, los dichos, metáforas, eslóganes, léxicos, glosarios, acrónimos, expresiones particulares y argot propio de la industria o sector. De igual modo considera el lenguaje no verbal o actitudinal como gestos, señales, expresiones corporales y posturas. Las historias son relatos verdaderos o elaborados sobre eventos que incitan a los individuos a adherirse a valores o acciones, resultando comunes los mitos (ficción) y los héroes-personificación de valores. (Allaire, 1992: 17).

Los rituales o rutinas sistemáticas y programas de la vida diaria indican y reafirman lo que es importante para la organización, así como el tipo de conducta esperado de sus miembros. Dentro de los rituales más conocidos 
se encuentran los sociales, como llamarse por el título; los laborales, como las formas de realizar trabajos; los directivos, como las reuniones para tomar decisiones, y los de reconocimiento, como la entrega de premios y distinciones. La conducta modelada incluye estándares, convenciones y costumbres que indican a los miembros la conducta más apropiada y el estilo de relación preferido - estándares idiomáticos, decoro público, trato y relaciones de amistad (Kennedy et al., 1982: 63-77).

En función de la visibilidad y la conciencia han sido establecidas tres capas de la cultura organizacional (Cardoso, 2008. págs. 105106): 1) Superficial y consciente (artefactos), en el que se encuentran todos los fenómenos vivibles, tangibles y audibles que ocurren como manifestación de los niveles más profundos; 2) Intermedio entre el consciente y el inconsciente (valores), formado por los conjuntos de principios que definen los artefactos; y 3) Invisible e inconsciente (supuestos), que determina las razones por las que un grupo percibe, piensa y siente de la manera como lo hace.

Otros estudiosos como Marcoulides y Heck (1993) identifican como dimensiones interrelacionadas de la $\mathrm{CO}$ : un sistema sociocultural del funcionamiento percibido de las estrategias y prácticas de la organización; un sistema de valor organizacional y las creencias colectivas de los empleados de la organización (en Yahyagil, 2006, pág. 209).

Como se dijo inicialmente, son múltiples los elementos culturales identificados, pero que en últimas subyacen a las dimensiones descritas anteriormente, entre otros se pueden mencionar: sistema de recompensas, decisiones de contratación, estructura gerencial, estrategia frente al riesgo, códigos, estereotipos, legados, leyendas, tradiciones, uniformes y modos de subversión. Otros autores incluso consideran el entorno o contexto, argumentando que es un factor que determina en buena medida lo que debe hacer la organización para ser exitosa.
Lo importante no es realizar un inventario de los distintos componentes, sino determinar su contribución en la construcción de un tipo particular de cultura.

\section{Tipos de cultura organizacional}

Aunque la clasificación en cualquier actividad social es un ejercicio riesgoso, conviene intentarlo desde el punto de vista analítico como única vía para comprender la complejidad de las dimensiones tratadas. De manera general, es importante señalar que los elementos positivos que operan en una organización y que originan una cultura integrada y compartida tienen sus raíces en cuatro agrupamientos institucionales básicos: la familia, las instituciones sociales, la comunidad científica y las instancias militares y legales (Truskie, 1999: 9-13). Estas instituciones enmarcan los cuatro modelos de $\mathrm{CO}$-relaciones humanas, sistema abierto, proceso interno y racional-, dando nacimiento a la siguiente tipología (Cameron and Quinn, 1999; Zammuto and Krakower 1991) $)^{4}$.

\subsection{Cultura grupal o de clan}

Inspirada en lainstitución familiary también denominada cultura de la cooperación. En ella, los miembros están comprometidos a colaborar $\mathrm{y}$ obtener resultados colectivos positivos, a observar los mandatos organizacionales basando su actuación en la confianza, la preocupación por los demás, la ayuda y un compartir permanente. Entre sus principales rasgos destacan la toma de decisiones participativa y la implementación a través de la construcción del consenso, así como la cohesión, el compromiso mutuo, la lealtad, la afiliación, la pertenencia, la cooperación, la calidez, la preocupación, la tradición, la moral y la equidad. El líder que guía esta cultura es un mentor involucrado y facilitador.

\subsection{Cultura adhocrática o de desarrollo}

Conocida también como cultura de la inspiración, emana fundamentalmente de las 
instituciones sociales. Los miembros creen fervientemente en los valores del humanismo, la responsabilidad social y el potencial individual, se motivan por la importancia de la tarea o por su apariencia ideológica. Caracterizada por su dinamismo y espíritu emprendedor, propensión al riesgo, innovación y desarrollo, crecimiento individual, recompensas intrínsecas, libre flujo de ideas e iniciativas, flexibilidad e individualidad, autocontrol, autogestión y empoderamiento. Sus líderes se distinguen por ser igualmente emprendedores, innovadores, creativos y arriesgados.

\subsection{Cultura jerárquica o burocrática}

Deriva principalmente de las organizaciones militares o de Policía y es denominada cultura consistente. En este tipo, se establecen pasos detallados, se programan los resultados esperados y se distribuyen los recursos necesarios para su cumplimiento. Existe una estandarización y rutinización de actividades, la información fluye verticalmente y las funciones se encuentran definidas de manera clara. Los individuos cumplen con mandatos organizacionales cuando los roles son formalmente asignados a través de reglas y regulaciones. Además de una inclinación hacia la medición, priman aspectos como las políticas, las estructuras formales, las recompensas basadas en rangos, la gestión de la información, la definición de roles, la estabilidad y la seguridad laboral. El líder jerárquico se diferencia por ser coordinador, organizador, conservador y precavido.

\subsection{Cultura racional u orientada al mercado}

Basada en los valores de la comunidad científica y ampliamente relacionada con la cultura del logro. Para garantizar el cumplimiento de sus propósitos fundamentales -productividad y eficiencia-, promueve a los individuos con base en las habilidades que demuestren para avanzar hacia nuevas teorías y tecnologías. Su aspiración central consiste en ganar clientes y consumidores ofreciendo productos, servicios, procesos y tecnologías acordes con las demandas del mercado. La colectividad que opera bajo este modelo se orienta hacia la producción, la competencia, el logro de metas y tareas, la investigación, el conocimiento y la información. Los líderes en esta cultura son netamente productores, directivos y enfocados hacia las metas.

A pesar de sus cualidades, los distintos modelos de cultura pueden coexistir en una misma organización, de hecho aquellas en las que domina un tipo particular son consideradas como disfuncionales. Sin embargo, en ocasiones las características y dinámicas de un sector o industria particular hacen que domine o prevalezca un tipo. Con base en el modelo de Cameron y Quinn(1999) es posible diseñar la figura 2 que esquematiza los tipos de $\mathrm{CO}$ en función de dos ejes básicos: el énfasis y la flexibilidad.

Figura 2

Tipos de cultura organizacional

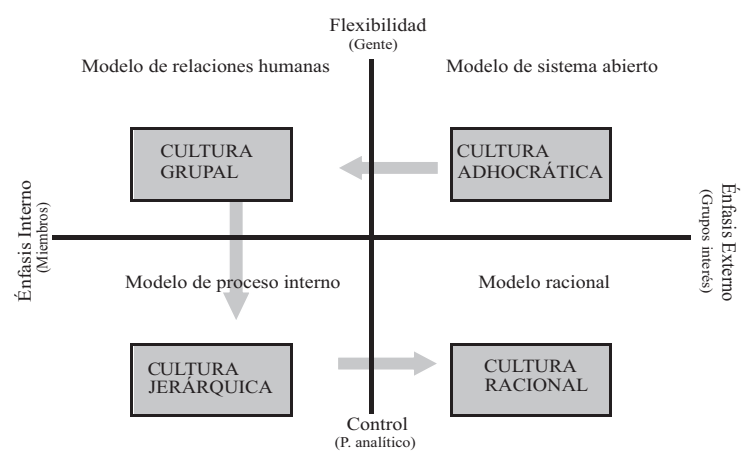

Fuente: Elaborado con información de Lisa Bradley et al., Bob Frew; Raymond F. Zammuto et al.

Mientras que el énfasis interno indica una marcada tendencia hacia el corto plazo, las actividades estables y la integración de unidades, para el externo el marco temporal es el largo plazo y su prioridad las actividades centradas en el logro, la diferenciación y la rivalidad. Por su parte, el eje superior señala la flexibilidad, la individualidad y la espontaneidad y el inferior la estabilidad, el control y la predictibilidad. Un análisis de los cuadrantes laterales permite observar que la cultura grupal y la jerárquica 
se inclinan hacia sus miembros y el presente, en tanto la adhocrática y la racional hacia los grupos de interés externos y el futuro. En este sentido, un movimiento hacia la derecha desde la cultura grupal o la jerárquica hacia la adhocrática o la racional representa mejoras en cuanto a la satisfacción del cliente, en tanto un desplazamiento hacia la izquierda implica una mayor preocupación por el empleado. Analizando los cuadrantes superiores e inferiores se advierte que la cultura grupal y la adhocrática presentan un marcado interés por la gente, mientras que a la jerárquica y a la racional incumbe el pensamiento analítico.

Según los estudios realizados, el tipo de cultura varía en función del tamaño de la organización - grande o pequeña-, del estado de su industria -madura o joven-y del sector en el que operen -público o privado-. A manera de ejemplo se tiene que por lo general las empresas pequeñas presentan una cultura grupal, aquellas pertenecientes a una industria madura tienen una racional, las que actúan en industrias jóvenes una adhocrática y las entidades gubernamentales por naturaleza presentan una jerárquica ${ }^{5}$. Es de anotar que estos resultados han sido observados en países como Estados Unidos, Inglaterra, Australia y España, por citar algunos, quedando pendiente entonces determinar si lo mismo ocurre en países en desarrollo, especialmente latinoamericanos, donde las condiciones en cuanto a tamaño y evolución de las industrias presentan marcadas diferencias.

Una preocupación permanente se ha centrado en identificar los rasgos idóneos que contribuyan al buen funcionamiento organizacional. Para los expertos resulta difícil precisar y prescribir el tipo perfecto de $\mathrm{CO}$, sin embargo, han avanzado en la determinación de cinco requisitos mínimos que garantizan no sólo la salud de la organización sino también la de sus miembros, ellos son: 1) desarrollar un sentido del accountability -rendición de cuentas- entre el personal y los directivos; 2) transmitir con coherencia los aspectos culturales; 3) pensar y actuar local y consistentemente; 4) estar sincronizados con los requerimientos competitivos del mercado mundial, y 5) adaptarse a las nuevas condiciones del mercado (Herbert, 2000: 151). De lo que se trata es de garantizar los atributos que puedan optimizar las probabilidades de éxito en un entorno competitivo.

Por último, frente al acentuado énfasis sobre los tipos de $\mathrm{CO}$ y sobre las posibilidades de que en una organización confluyan varios de ellos, surgen dos interrogantes ¿Qué modelos coexisten o dominan? ¿En qué proporción se presentan? Para dar solución a estos cuestionamientos se deben conocer y explorar de antemano las distintas metodologías disponibles para la medición de la CO.

\section{4. ¿Es posible medir la cultura organizacional?}

Si bien los estudios de $\mathrm{CO}$ emergen de una combinación de la psicología organizacional, la psicología social y la antropología social, durante los últimos tiempos han cobrado mayor significado para otras disciplinas, dados los efectos de la cultura sobre todo tipo de acción colectiva. Esto ha generado otro debate importante: la metodología para estudiarla. Frente a la supuesta dificultad para cuantificar factores como valores, creencias y prácticas institucionales, los esfuerzos se han centrado en los diagnósticos para identificar las distintas dimensiones culturales.

Para precisar el modelo de CO se dispone de dos enfoques fundamentales, el cualitativo y el cuantitativo. Estas dos alternativas, además de tener diferentes orientaciones, proporcionan variados puntos de influencia para el entendimiento de la CO. Por ello, la decisión sobre el tipo de estudio dependerá de su efectividad para: determinar la magnitud en que las organizaciones exhiben culturas fuertes; predecir relaciones entre el tipo cultural y otros fenómenos; y mapear perfiles culturales 
(Zammuto y Krakower, 1991: 84-87). En últimas, la elección de un enfoque cuantitativo o cualitativo dependerá en buena medida de los objetivos planteados en el estudio.

La caracterización cualitativa de la $\mathrm{CO}$, por lo regular, ofrece como resultado una distinción binaria: 1) una cultura fuerte, diferenciada porque proporciona un claro sentido de identidad con el personal y porque todos conocen las metas organizacionales y trabajan por ellas, y 2) una cultura débil, es decir, carente de valores y prácticas bien definidas y con poca influencia en la conducta del empleado, por lo que en últimas prefieren abandonarla. Una valoración de este tipo, desde luego, ofrece una visión sobre la $\mathrm{CO}$, no obstante, es imposible determinar la magnitud de una condición específica, resultando un tanto ambigua y poco confiable en el caso de demandar algún tipo de intervención ${ }^{6}$.

Dentro de la gran cantidad de estudios cualitativos realizados, destacan los etnográficos, cuyo fin primordial es descubrir la manera como los actores dan un significado a los acontecimientos y a las acciones del mundo organizacional. El trabajo etnográfico implica una relación participativa entre el individuo que trata de comprender (investigador) y los sujetos del estudio (miembros), su estrategia central es el diálogo y no la observación (Abravanel, 1988: 134).

Los estudios etnográficos, al igual que otros cualitativos, permiten una profundización en el estudio de la cultura en la medida que se obtienen percepciones estructuradas de los propios sujetos y sobre la forma en que se adaptan a los cambios internos. Privilegian, de igual modo, el conocimiento de intereses, vivencias, motivaciones y elementos encubiertos e invisibles. Dentro de los estudios cualitativos más utilizados se identifican: el Focus Group, desarrollado para descubrir tanto los motivadores de los distintos colectivos así como los insatisfactores; la Percepción de Valores y
Filosofías, que mediante la aplicación de una encuesta estructurada facilita el entendimiento de los principales valores percibidos; los Juegos Proyectivos, que a través del desarrollo de actividades lúdicas identifica el perfil de cultura organizacional; las Entrevistas a Profundidad, que permiten obtener información para elaborar un diagnóstico acerca de los procesos desarrollados; y, por último, el Intercambio de Roles, que identifica mediante dinámicas las dificultades o facilidades de los miembros de una organización para entender las necesidades y expectativas de grupos de interés externos?

En algunos países como Brasil, los estudiosos de la cultura han optado por crear instrumentos cualitativos ajustados a su propio contexto. En la categoría de instrumentos para la validación de cultura organizacional se encuentran las escalas desarrolladas por Gomide Jr. E Martins (1997) a partir de los cuatro tipos de cultura de Handy (club, función, tarea y existencial). Tamayo y Godim (1996) construyeron una escala con la intención de verificar una estructura de 38 valores de las organizaciones, distribuidos en cinco factores o dimensiones relacionados con la eficacia y la eficiencia organizacional (Ferreira, 2002. págs. 4-5).

Actualmente, algunos académicos y varios policymakers muestran especial inclinación por la medición cuantitativa de la cultura, dado su interés en determinar la relación de esta con el desempeño y la calidad en las organizaciones. Es así como, durante las últimas dos décadas, una gran cantidad de herramientas han sido diseñadas para medir la $\mathrm{CO}$, especialmente en ámbitos como el gubernamental, el educativo y el hospitalario. Estos instrumentos dan cumplimiento a tres requisitos que han sido establecidos como básicos para garantizar un rigor en la investigación y una solidez en los hallazgos, ellos son: 1) ser cuantitativo de preferencia o semicualitativo;2) denotar eficacia para evaluar un amplio rango de dimensiones culturales, y 3) ofrecer posibilidades válidas y confiables para el procesamiento estadístico de los datos (Scott, 2003: 2). 
Las herramientas para la medición de la $\mathrm{CO}$ se clasifican de acuerdo con cinco aspectos fundamentales a saber: 1) el enfoque, que puede ser tipológico, cuyo propósito es evaluar y obtener como resultado uno o más tipos de cultura o dimensional, que describe una cultura por su posición frente a cierto número de variables continuas; 2) la fundamentación, sea esta teórica con una fuerte derivación conceptual o pragmática en la que el investigador se sumerge en la cultura a través de una observación profunda; 3) el alcance de la evaluación, que puede ser en torno a una dimensión específica de la $\mathrm{CO}$ o a un rango de dimensiones; 4) el potencial para explorar con relación al clima organizacional -percepciones y opiniones del personal sobre su ambiente de trabajo-, o a la cultura organizacional -manifestaciones profundas como los valores y las creencias-, y 5) la magnitud de su uso en estudios empíricos, que varía en función del grado de validez y confiabilidad, así como de los métodos usados para evaluar sus propiedades científicas (2003: 3).

Dentro de los instrumentos cuantitativos más usados se pueden citar: el Cuestionario de la Ideología Organizacional de Harrison (Harrison's Organization Ideology Questionnaire, 1972), el Modelo de Valores en Competencia (Competing Values Framework, 1981), el Inventario de Cultura Organizacional (Organizational Culture Inventory, 1987), el
Cuestionario de Cultura Hospitalaria (Hospital Culture Questionnaire 1993), el Cuestionario de Cultura de MacKenzie (MacKenzie's Culture Questionnaire, 1995), la Encuesta de Calidad de la Cultura y Clima Organizacional (Quality Culture and Organizational Climate Survey), el Cuestionario de Prácticas Culturales (Practice Culture Questionnaire, 2000) y el Cuestionario de Cultura Corporativa (Corporate Culture Questionnaire). Se ha demostrado de manera contundente que estas herramientas han sido y pueden ser ajustadas, de acuerdo con las demandas y necesidades de los investigadores. Así mismo que la selección de una de ellas depende principalmente del propósito de la investigación, del uso pretendido de los resultados y de la disponibilidad de recursos.

La mayoría de herramientas utilizadas en los estudios sobre CO se basan -dada su validez, confiabilidad y eficacia- en el Modelo de Valores en Competencia (MVC), desarrollado originalmente por Cameron y Quinn a partir de los Arquetipos Psicológicos de Jung. Este modelo permite explorar estructuras profundas de la $\mathrm{CO}$, en torno a aspectos como las características dominantes, el liderazgo organizacional, la gerencia del personal, los valores, el énfasis estratégico y los criterios del éxito. Los rasgos de cada uno de los tipos de $\mathrm{CO}$ en función del MVC pueden apreciarse en la tabla 1.

Tabla 1

Modelo de los valores en competencia

\begin{tabular}{|c|c|c|c|c|}
\hline Aspectos & Cultura grupal & Cultura adHOCrÁtica & Cultura JerÁRQuica & Cultura racional \\
\hline $\begin{array}{l}\text { Características } \\
\text { dominantes } \\
\text { Organización }\end{array}$ & Lugar personal y familiar & $\begin{array}{l}\text { Muy dinámica y } \\
\text { emprendedora }\end{array}$ & $\begin{array}{l}\text { Jerarquizada, controlada } \\
\text { y estructurada }\end{array}$ & $\begin{array}{l}\text { Orientada hacia los } \\
\text { resultados }\end{array}$ \\
\hline Gente & $\begin{array}{l}\text { Comparten mucho con los } \\
\text { demás }\end{array}$ & $\begin{array}{l}\text { Dispuesta a apostar por } \\
\text { sus ideas y a asumir riesgos }\end{array}$ & $\begin{array}{l}\text { Gobernada por } \\
\text { procedimientos y normas } \\
\text { formales }\end{array}$ & $\begin{array}{l}\text { Competitiva, orientada } \\
\text { al logro de objetivos }\end{array}$ \\
\hline $\begin{array}{l}\text { Liderazgo } \\
\text { organizacional }\end{array}$ & $\begin{array}{l}\text { Tutor, consejero y padre } \\
\text { de todos en la familia }\end{array}$ & $\begin{array}{l}\text { Emprendedor nato, } \\
\text { innovador } \\
\text { y tomador de riesgos }\end{array}$ & $\begin{array}{l}\text { Coordinador, organizador } \\
\text { y defensor de la eficiencia }\end{array}$ & $\begin{array}{l}\text { Ejecutivo agresivo, } \\
\text { competitivo, clara } \\
\text { orientación hacia los } \\
\text { resultados }\end{array}$ \\
\hline
\end{tabular}




\begin{tabular}{|l|l|l|l|l|}
\hline \multirow{2}{*}{ Estilo gerencial } & $\begin{array}{l}\text { Promueve el trabajo } \\
\text { en equipo, consenso, } \\
\text { participación }\end{array}$ & $\begin{array}{l}\text { Promueve la iniciativa del } \\
\text { individuo, la adopción de } \\
\text { riesgos, la innovación, la } \\
\text { libertad y la singularidad }\end{array}$ & $\begin{array}{l}\text { Promueve la seguridad } \\
\text { del empleo, la } \\
\text { estabilidad y predicción }\end{array}$ & $\begin{array}{l}\text { Competitividad } \\
\text { agresiva, logro de } \\
\text { objetivos ambiciosos }\end{array}$ \\
\hline \multirow{2}{*}{$\begin{array}{l}\text { Valores } \\
\text { organizacionales }\end{array}$} & $\begin{array}{l}\text { Lealtad, compromiso } \\
\text { organizacional, } \\
\text { confianza mutua }\end{array}$ & $\begin{array}{l}\text { Compromiso con la } \\
\text { innovación y cambio } \\
\text { continuo }\end{array}$ & $\begin{array}{l}\text { Respeto por normas } \\
\text { y políticas formales, } \\
\text { cumplimiento con la } \\
\text { jerarquía, coordinación }\end{array}$ & $\begin{array}{l}\text { Agresividad, espíritu } \\
\text { ganador, consecución } \\
\text { de objetivos }\end{array}$ \\
\hline Énfasis estratégico & $\begin{array}{l}\text { Desarrollo humano } \\
\text { de los miembros, } \\
\text { confianza, mentalidad } \\
\text { abierta }\end{array}$ & $\begin{array}{l}\text { Dinamismo y } \\
\text { predisposición a aceptar } \\
\text { nuevos retos; experimentar } \\
\text { nuevas cosas; aprender de } \\
\text { los errores }\end{array}$ & $\begin{array}{l}\text { fe sus miembros, } \\
\text { ficiencia, control y } \\
\text { funcionamiento fluido }\end{array}$ & $\begin{array}{l}\text { Acciones competitivas, } \\
\text { vencer a la competencia }\end{array}$ \\
\hline Criterios del éxito & $\begin{array}{l}\text { Desarrollo humano, } \\
\text { trabajo en equipo, } \\
\text { compromiso e interés } \\
\text { por los trabajadores }\end{array}$ & $\begin{array}{l}\text { Desarrollo de productos } \\
\text { únicos y novedosos }\end{array}$ & $\begin{array}{l}\text { Eficiencia, confiabilidad } \\
\text { en el servicio, adecuada } \\
\text { programación de la } \\
\text { producción, costos bajos }\end{array}$ & $\begin{array}{l}\text { Penetración y } \\
\text { liderazgo en el } \\
\text { mercado }\end{array}$ \\
\hline
\end{tabular}

Fuente: Tomado de Cameron y Quinn (1999)

Algunos estudios -especialmente en el sector educativo- han considerado la aplicación de dos instrumentos, uno para caracterizar el modelo de cultura y otro para identificar el tipo de cultura ideal de acuerdo con la percepción de los miembros de la organización. La imagen de la cultura ideal es valorada mediante un instrumento secundario denominado Imágenes de las Escuelas a través de la Metáfora (Images of Schools Through Metaphor ISM) que formula dos imágenes en función de metáforas, de modo tal que el personal proyecta tanto la situación actual como la ideal. Los datos de la Encuesta de Desempeño Institucional (Institutional Performance Survey IPS) y de las ISM apoyan la formulación de estrategias orientadas a cambiar y/o reforzar la cultura existente (Frew, 1996: 4-10).

Cuando los estudios tienen considerada una etapa de intervención, es recomendable la aplicación de dos o más instrumentos que ofrezcan el nivel de detalle requerido. Frente a esto resulta conveniente una triangulación de herramientas -enfoque multimetódico-, de este modo, un cuestionario cuantitativo que examina valores y creencias puede ser complementado con técnicas cualitativas como la observación para explorar manifestaciones superficiales o la entrevista para indagar a profundidad supuestos implícitos. Si bien es cierto que la mayoría de instrumentos han sido aplicados en el campo de la educación, de la salud y de las actividades gubernamentales, es responsabilidad de los investigadores identificar y ajustar aquellos que presenten algún potencial para ser usados en otros ámbitos.

Finalmente, cabe señalar que los estudios cuantitativos de la $\mathrm{CO}$ resultan útiles para examinar las relaciones de tipo cultural y su fortaleza con otros fenómenos tales como la efectividad de la organización y su adaptación a las condiciones cambiantes del entorno. Si bien las investigaciones cualitativas proporcionan una visión sobre estas relaciones, es un tanto difícil y arriesgado generalizar resultados (Zammuto y Krakower, 1991: 110). 


\section{El impacto de la cultura en el desempeño organizacional}

A pesar de que siempre está presente, la cultura es fácilmente ignorada desconociendo así su impacto en la efectividad organizacional. Tradicionalmente, la efectividad fue definida en torno al logro de las metas, enfoque que dominaron por décadas los estudios organizacionales. Por fortuna, en la actualidad se cuenta con una serie de enfoques alternativos que van más allá del logro de las metas y consideran aspectos cruciales como los recursos, los procesos internos, los stakeholders y los valores en competencia. La efectividad organizacional ha sido entendida como la satisfacción neta de todos los stakeholders en el proceso de transformación de insumos en productos de manera eficiente, la utilidad debe ser para todas las partes involucradas (Zammuto, 1984 en Kowalski et al., 2005. pág. 252).

Las medidas del desempeño pueden dividirse en dos grupos: las financieras, que carecen de flexibilidad e ignoran las demandas de los clientes (utilidades, retorno de la inversión, variación de precios y productividad), y las no financieras (Saad et al. 2006. pág. 40). Los criterios para medir la efectividad pueden ser generales y amplios -supervivencia y utilidad- o estrechos y basados en funciones particulares -niveles jerárquicos, roles, procesos-. Campbell (1977) por ejemplo, ha identificado cerca de 30 criterios para medir la efectividad, que van desde la satisfacción laboral hasta el crecimiento y la productividad. Como ya se mencionó en el MVC, a cada cultura corresponden unos criterios propios de efectividad, de modo tal que para la tipología grupal cuenta el compromiso, la cohesión y la moral; para la adhocrática, la adaptabilidad y proactividad; para la jerárquica, la estabilidad y la continuidad; y para la racional, la productividad y la eficiencia. Es así como se reconoce esta herramienta como apropiada para determinar el impacto de la cultura en la actuación de las organizaciones, pues combina diversos indicadores de efectividad y desempeño (Grant, 2003: 6).

No cabe duda de que la CO ostenta la clave para entender el desempeño pasado y para predecir la productividad futura (Joyner, 2001: 26). Por tanto, no se debe desconocer su contribución al éxito de las organizaciones, independientemente de que se pueda determinar en qué medida lo hace ${ }^{8}$. La noción de éxito varía en función del contexto económico, social y cultural de los países. En Japón, por ejemplo, el atributo más notable es la preocupación por las necesidades sociales y ambientales; en Italia lo es la efectividad del enfoque de administración y gerencia: en México lo es la estabilidad y la rentabilidad (Herbert, 2000: 151).

La productividad, por su parte, también constituye uno de los referentes para medir el éxito, especialmente en nuestros países. En consonancia, la solución para aumentar la productividad no es copiar modelos, teorías o enfoques administrativos y gerenciales, sino luchar por entender y fortalecer la CO. Muchos se preguntan acerca de la relación o vínculo existente entre la cultura y la productividad de una organización, la respuesta a este interrogante es sencilla. En la medida que se cuente con una cultura fuerte, sus miembros conocerán qué se espera exactamente de ellos, por lo que gastarán menos tiempo en decidir cómo actuar frente a una situación determinada. De igual modo, removerá en buena parte la incertidumbre del personal ya que proporciona además de una estructura, estándares y un sistema de valores para operar (Kennedy et al., 1982: 15-16).

Algunos estudiosos han logrado identificar dos dimensiones básicas para determinar la relación de la cultura y el desempeño organizacional, ellas son: 1) las dimensiones de contenido o aspectos que capturan la esencia de la $\mathrm{CO}$ y reflejan lo que es la organización frente al enfoque-interno o externo-, a la velocidad, al riesgo, a la participación, a la claridad, al poder y al individualismo; y 2) las dimensiones modelo, 
que ofrecen un resultado de las interrelaciones entre las anteriores en función de la fortaleza, la congruencia y el tipo culturales. Sobre esta última dimensión, autores como Nadler and Tushman (1980); Deal and Kennedy (1982), y Cameron and Ettington (1988), consideraron sus factores como fuertes predictores de la efectividad. La fortaleza se refiere al dominio o preeminencia de ciertos aspectos de la cultura que afectan todo lo que pasa internamente. La congruencia concierne al grado en el que la cultura de una parte de la organización es similar y consistente con la reflejada en otra parte. El tipo enfatiza el patrón específico de cultura manifiesto, es decir, la medida en que ciertos atributos culturales o dimensiones de contenido dominan otros (National Research Council Staff, 1997: 73-74).

Algunas corrientes de investigación se han dado a la tarea de desarrollar modelos explícitos de cultura organizacional y efectividad, así como métodos para sumedición, basados en cuatrorasgos culturales de efectividad de las organizaciones. Fey y Denison (2003: 688) compilan la caracterización de estos atributos como sigue: 1) Involucramiento: las organizaciones efectivas facultan a la gente, organizan equipos y desarrollan capacidades humanas (Becker, 1964; Lwler, 1996; Likert, 1961). La gente está comprometida y siente un fuerte sentido de pertenencia, participan en las decisiones y conectan sus metas individuales con las de la organización (Katzenbach, 1993; Spreitzer, 1995). 2) Consistencia: las organizaciones efectivas cuentan con normas del comportamiento cimentadas en valores esenciales y con líderes y seguidores capaces de lograr acuerdos (Block, 1991). La consistencia es una fuente de estabilidad e integración interna, producto de formas de pensar comunes. 3) Adaptabilidad: las organizaciones integradas son a menudo las menos sensibles (Kanter, 1983). En este sentido, la integración interna y la adaptación externa pueden estar en desacuerdo. Las organizaciones adaptables son conducidas por sus clientes, toman riesgos y aprenden de sus errores, tienen la capacidad y experiencia en generar el cambio (Nadler, 1998; Senge, 1990; Stalk, 1988). 4) Misión: las organizaciones efectivas tienen un claro sentido del propósito y dirección, definen metas y objetivos estratégicos y expresan una visión del futuro (Mintzberg, 1987, 1994; Ohmae, 1982; Hamel y Parlad, 1994).

Los autores demostraron que los anteriores atributos estaban relacionados con diversos criterios de efectividad, encontrando que rentabilidad estaba altamente correlacionada con misión y consistencia, innovación con involucramiento y adaptabilidad y crecimiento en ventas con adaptabilidad y misión. Las organizaciones efectivas serán aquellas capaces de resolver las contradicciones entre integración interna y adaptabilidad externa (Fey y Denison 2003: 688).

La cultura es un determinante del desempeño ya que impacta no sólo los resultados directos y la eficiencia de una organización, sino también la salud, el entusiasmo, el compromiso y la flexibilidad de su personal. Bajo estas circunstancias entenderla, darle la atención necesaria y administrarla, facultará a los líderes para maximizar el capital intelectual, actitudinal y conductual de su gente (Darcey-Lymm et al., 2003). No se puede dejar de considerar que así como el líder incide directamente en la cultura, la cultura de una organización también es responsable de definir el perfil del líder, es decir al tiempo que moldea es moldeado (Woida et al. 2006. pág. 48). La CO, pues, establece el escenario para la determinación de la estrategia y mejora los aspectos operacionales de la vida organizacional (pág. 37). Aparece como concepto básico para entender fenómenos sociales en las organizaciones y para explicar diferencias entre compañías, particularmente aquellas que operan en el mismo mercado (Salaman. 2000. p. 37).

\section{Conclusiones}

Revisados los asuntos atinentes a la cultura organizacional es difícil desconocer su importancia para la interpretación de la 
vida, para el comportamiento organizacional, así como para la comprensión de la capacidad de adaptación de los individuos al cambio, aspectos centrales en los procesos de acción colectiva. La disonancia o incompatibilidad entre la cultura y el sistema socioestructural constituye, por tanto, un aspecto a considerar ya que explica, entre otras, la pérdida de efectividad $\mathrm{y}$ el deterioro en el funcionamiento de las colectividades. El presente trabajo pretendía conceptuar en torno a la Cultura Organizacional $\mathrm{CO}$, sus dimensiones y componentes, así como identificar los tipos y enfoques para su medición, para en última instancia determinar su impacto sobre el desempeño organizacional. Corresponde entonces destacar los desarrollos más importantes al interior de cada apartado.

Las definiciones de CO además de numerosas, resultan complejas y divergentes; todos saben qué es pero pocos son capaces de identificarla y explicarla. Más aún, muchos son los que la invocan, especialmente en el medio latinoamericano, y pocos quienes realizan propuestas concretas que permitan su caracterización y medición. Si bien las definiciones son variadas, todas reflejan unos elementos comunes como: mandatos aprendidos, reglas no escritas, supuestos compartidos, valores y prácticas compartidas, conducta aceptable, modo correcto y un moldear de la conducta humana. Conceptos que en conjunto evidencian la informalidad compartida que gobierna y legitima la conducta humana. En muchas organizaciones, incluidas las entidades gubernamentales, la preocupación actual se concentra en el personal, al considerarlo el activo más valioso, apreciación un tanto desfasada, por cuanto lo que verdaderamente define el carácter de toda organización es su cultura y no su gente.

Tres son las dimensiones que agrupan los diversos componentes de la $\mathrm{CO}$, una considerada visible o manifiesta y otras dos invisibles, la estratégico y la esencial. La primera referida al conjunto de prácticas institucionales, la segunda a las convicciones y certezas de los líderes y la última a las premisas y preceptos compartidos. La identificación e inventario de elementos de la CO no resultan suficientes, lo importante es determinar la contribución de cada uno al complejo cultural de la organización.

De igual manera, se han identificado cuatro agrupamientos que enmarcan los modelos de $\mathrm{CO}$, son ellos la familia, las instituciones sociales, la comunidad científica y las instancias militares y legales. Modelos que dan origen a los tipos de cultura: grupal -de clan o de cooperación-, entre cuyos miembros prima el compromiso, la confianza y la solidaridad; adhocrática-de desarrollo o de inspiración-, en la que predominan los valores del humanismo y la responsabilidad social; jerárquica -burocrática o consistente-, caracterizada por la estandarización de actividades y la obediencia de mandatos organizacionales; y la racional -orientada al mercado o del logro-, en cuyo interior se reconocen habilidades y se privilegia la generación de conocimiento e información. Si bien son cuatro tipos de cultura, debe evitarse el encasillamiento de las organizaciones, ello es asimilar siempre a las empresas como una cultura racional, a las entidades gubernamentales como jerárquica, a las no gubernamentales como adhocrática y a las cooperativas como grupal, puesto que en cualquiera de ellas pueden coexistir los cuatro tipos y puede, incluso, dominar o prevalecer un tipo sobre los demás.

Para determinar el modelo de $\mathrm{CO}$ existen dos posibilidades, los enfoques cualitativos y los cuantitativos, la elección depende de los objetivos perseguidos, ello es caracterización, medición o intervención. Los estudios cualitativos más comunes son los etnográficos, aunque no se deben desconocer otros como el Focus Group, la Percepción de Valores y Filosofías, los Juegos Proyectivos, las Entrevistas a Profundidad y el Intercambio de Roles, por mencionar algunos. De otra parte, puede afirmarse que los policymakers 
son precursores en la aplicación de enfoques cuantitativos para la medición de la CO. Muchos de ellos han validado instrumentos en el ámbito gubernamental - hospitalario y educativo-, usando como base fundamental el Modelo de los Valores en Competencia, de Cameron y Quinn.

Es innegable el impacto de la cultura en la efectividad de las organizaciones, entendida esta en términos de satisfacción, crecimiento, productividad, responsabilidad social y medioambiental, estabilidad, rentabilidad, cobertura, etc., dependiendo de sus actividades y ámbito de operación. Pero crear una cultura no es tarea fácil, requiere una inversión constante y sustancial de recursos, especialmente tiempo, sin embargo, puede traer enormes beneficios a través de la creación de relaciones a largo plazo tanto internas como externas. Muchas organizaciones crean culturas para sobrevivir y no para operar efectivamente, funcionan sobre la base de relaciones transitorias con sus stakeholders por lo que nunca podrán garantizar una verdadera confianza de sus miembros, sociedad y demás públicos de interés (Emmons, 2004: 2).

La cultura es la responsable de mantener la estructura social en la organización, además de generar la identidad de esta y la diferencia de otras. Sus distintas capas son mantenidas y transmitidas a través de procesos de socialización entre los empleados (Kwantes, 2007. pág. 98). A pesar de que al interior de las ciencias administrativas se ha hablado mucho de la cultura organizacional, quienes han realizado importantes aportes para la medición de la misma son los estudiosos de las ciencias sociales. El reto, por tanto, es para las primeras si desean dar el salto hacia la medición de la cultura en las unidades productivas o empresariales. Finalmente, es importante llamar la atención sobre la necesidad de estudiar los asuntos culturales del sector gubernamental en Latinoamérica, a partir de la realidad de sus países que a todas luces evidencia una demanda por un mejor desempeño de su función pública.
Indudablemente, la consideración de la cultura organizacional en el accionar gubernamental contribuirá al ya viejo propósito del buen gobierno.

\section{Notas}

${ }^{1}$ Aunque instituciones y organizaciones a menudo se presentan como sinónimos, conviene hacer la respectiva distinción. Mientras las primeras son reglas formales e informales capaces de moldear la conducta de individuos y organizacionesenunasociedad.Lasorganizaciones son entes compuestos por individuos que actúan colectivamente para lograr objetivos compartidos. De este modo, organizaciones e individuos persiguen sus intereses dentro de una estructura institucional definida por reglas formales (constituciones, leyes, regulaciones, contratos) e informales (ética, preceptos religiosos, códigos de conducta implícitos). Las organizaciones tienen reglas internas para restringir la conducta de sus miembros. Las instituciones representan la estructura que incentiva la conducta de organizaciones e individuos (Burki (1998) et al.. pág. 16).

${ }^{2}$ Para ampliar respecto a la etimología y génesis del término cultura, remitirse a Chan (2001).

3 Una interesante presentación de los debates surgidos en torno al concepto de cultura organizacional es presentado por Dávila y Martínez (1999).

${ }^{4}$ Ouchi (1981) y Wilkins y Ouchi (1983) caracterizaron la cultura organizacional en mercados, jerarquías y clanes cuyas formas de control son los mecanismos de precios, las relaciones de autoridad y los valores y creencias compartidos, respectivamente (Wilkins y Ouchi, 1983; Ouchi, 1979, 1980, 1981, en Bates et al, 1995. pág. 1.568).

${ }^{5}$ Para estos efectos se recomienda una revisión completa de las conclusiones del estudio de Zammuto y Krakower (1991). 
6 Muchos son los calificativos usados para caracterizar la cultura, por lo que es común oír hablar en términos positivos de una cultura rica, coherente, cohesiva, significativa, vibrante, centrada, integrada o balanceada; en términos negativos de una fragmentada, pobre, poco entendida, depredadora, congelada, caótica, confusa, fría, malsana y difusa, entre otros. Sin importar el calificativo, el éxito en una organización dependerá de la lectura adecuada de la cultura y de la habilidad para perfeccionarla y moldearla de acuerdo con las circunstancias cambiantes del entorno.

${ }^{7}$ Para ampliar en torno a instrumentos cualitativos consultar Baraona B. (2005).

8 Las dificultades para determinar el impacto de la cultura en el desempeño de las organizaciones obedecen a que no se tiene una clara definición de lo que significa el éxito para cada una.

\section{Referencias}

Abravanel, H. (1988), "En busca de los mitos organizacionales" en Abravanel, H. et al. Cultura organizacional. Aspectos teóricos, prácticos y metodológicos. Bogotá: Legis.

Albrow, M. (1999). Sociology: The Basics. Londres: Routledge.

Allaire, Y. \& Michaela E. F. (1992). “Teorías sobre la cultura organizacional" en Abravanel et al., Cultura organizacional. Aspectos teóricos, prácticos y metodológicos. Bogotá: Legis.

Alvesson, M. (2002). Understanding Organizational Culture. Londres: Sage Publications.

Barahona, R. (2005). Diagnóstico de la cultura de empresa en la caja de ahorros de
Guadalajara. [Tesis doctoral]. Madrid: Universidad Complutense de Madrid.

Bates, K. A. et al. (1995, oct). "The Crucial Interrelationship between Manufacturing Strategy and Organizational Culture" en Management Science, Vol. 41, N. ${ }^{\circ} .10$. págs. 1565-1580.

Bradley, L. \& Parker R. (2001), “Organisational culture in the public sector". Paper.

Brinton, Mary C. \& Nee V. (Edit.) (2001), New Institutionalism in Sociology. Palo Alto: Stanford University Press.

Burlingham, B. (2001). "What's your culture worth”, Inc. págs. 124-133.

Burki, S. J. \& Perry, G. E. (1998). Beyond the Washington Consensus: Institutions Matter. Washington DC: World Bank.

Cameron, K. S. \& Quinn, R. E. (1999). Diagnosing and changing organizational culture. Nueva Jersey: Prentice-Hall.

Cardoso, M. \& Alves, P. (2008, feb.). "Uma reflexão sobre a cultura organizacional à luz da Psicanálise". Revista Brasileira de Enfermagem. 61(1): 103-108.

Chan, A. (2001). Toward a genealogy of organizational culture. The perspectives of Foucault. Filadelfia: John Benjamins Publishing Co.

Darcey-Lymm M. \& S. Farbrother (2003, oct.). "Changing organization culture one face at a time" en Public management. V 85 i9 pág. 14(4).

Dávila, A. \& Martínez, N. (1999). “Un acercamiento crítico al concepto de cultura organizacional: implicaciones para su estudio en organizaciones latinas". 
En Anabella Dávila y Nora H. Martínez (Coord.), Cultura en Organizaciones Latinas. México: Siglo XXI editores.

Emmons, P. (2004, julio). "Growing smart: achieving success with a well-defined corporate culture" en Concrete construction.

Ferreira, M. C. (2002). "Desenvolvimento de um instrumento brasileiro para avaliação da cultura organizacional". En Estudos de Psicología. (Natal), Vol. 7, No. 2. págs. 271-280.

Fey, C. F. \& Denison, D. R. (2003). "Organizational Culture and Effec-tiveness: Can American Theory Be Applied in Russia?" en Organization Science, Vol. 14, No. 6, págs. 686-706.

Fitzgerald, V. (Ed.) (2002). Social Institutions and Economic Development. A Tribute to Kurt Martin. Secaucus: Kluwer Academic Publishers.

Frew, B. (1996). "The assessment of organizational culture at the Sidney Institute of Technology" [en línea] disponible en http://www.aare.edu.au/96 pap/frew 96179.txt, recuperado: julio de 2007.

Grant, G. (2003). ERP \& data warehousing in organizations: Issues and challenges, Hershey: Igi Global.

Gray, C. (1998). "The enterprise culture model of development" en Enterprise. Florence, KY, USA, Routledge.

Hannan, M. T. et al. (2003, sept.). "The Fog of Change: Opacity and Asperity in Organizations", en Administrative Science Quarterly, Vol. 48, N. ${ }^{\circ} 3$, págs. 399-432.
Herbert, G. (2000) "Corporate culture. Great businesses turn on a little pin" en Mitchell Charles, A short course in international business culture. Novato: World Trade Press.

Jenks, Ch. (1993). Culture. London: Routledge.

Joyner, J. (2001). "Corporate culture defines success" en Computing Canada, V. 27 i11.

Kennedy, A. A. \& Deal T. E. (1982). Corporate culture. The rites and rituals of corporate life. USA: Adiison-wesley publishing Co.

Kowalski,K.B.\&Swanson,J.A.(2005). “Critical success factors in developing teleworking programs", en Benchmarking: An International Journal. Vol. 12, núm. 3. págs. 236-249.

Kwantes, C. T. (2007). "Organizational culture fit and outcomes in six national contexts: an organizational level analysis". En Journal of Organizational Culture, Communications and Conflict, Vol. 11, No. 2. págs. 95-111.

Lau, Chung-Ming, Tse, D. K. \& Zhou, Nan. (2002, sept.-dic.). "Institutional Forces and Organizational Culture in China: Effects on Change Schemas, Firm Commitment and Job Satisfaction", en Journal of International Business Studies, Vol. 33, N. ${ }^{\circ} 3$. págs. 533-550.

Lundberg, C. (2000). "Knowing and surfacing organizational culture. A consultant's guide" en Robert T. Golembiewski (Edit.), Handbook of organizational consultation. Págs. 701-713. New York: Marcel Dekker Incorporated.

National Research Council Staff (1997). Enhancing organizational performance. Washington: National Academies Press. 
North, D. C. (1993), Instituciones, cambio institucional y desempeño económico. México: Fondo de Cultura Económica.

Rowlinson, M. \& Procter, S. (Verano, 1999). "Organizational Culture and Business History”, en Organization Studies. 26p.

Pires, J. C. \& Macedo, K. (2006) "Cultura organizacional em organizações públicas no Brasil", en Revista de Administração Pública [online]. Vol. 40, No. 1.

Saad, M. \& Patel, B. (2006). “An investigation of supply chain performance measurement in the Indian automotive sector", en Benchmarking: An International Journal. Vol. 13 No. 1/2. págs. 36-53.

Salaman, Graeme (Ed.) (2000). Understanding Business: Organisations. London: Routledge.

Scott, T. (2003). "The quantitative measurement of organizational culture in health care: A review of the available instrumentsmethods" en Health Services Research.

Shepsle, K., (1999). "La economía Política de la Reforma Estatal: política hasta la medula" en Gandour, M. et al., Hacia el rediseño del Estado. Análisis Institucional, reformas y resultados económicos. Bogotá: Tercer Mundo.

Schein, E. H. (1988). La cultura empresarial y el liderazgo. España: Plaza \& Janes.

The Economist (2002), "When something is rotten; corporate culture (Creating a healthy corporate culture)".

Truskie, S. D. (1999). "The $\mathrm{L}^{4}$ strategy: forming a performance-enhancing culture" en Leadership in high-performance organizational cultures. Wesport: Greenwood Publishing Group Incorporated.
Urrea, F. (2000). Innovación y cultura de las organizaciones en tres regiones de Colombia. Bogotá: Colciencias y Corporación Calidad.

Vaitsman, J. (2000). "Cultura de organizações públicas de saúde: notas sobre a construção de um objeto", en Cadernos de Saúde Pública, Vol. 16, No. 3, págs. 847-850.

Want, Jerry. (2003). "When worlds collide: culture clash, corporate cultureilluminating the black hole" en Journal of Business Strategy. Vol. 24 i4. pág. 14(8).

Weber, R. A. \& Camerer, C. F. (2003, abril). "Cultural Conflict and Merger Failure: An Experimental Approach", en Management Science, Vol. 49, No. 4, págs. 400-415.

Woida, L. M., Vieira, J. V. \& Pomim, M. L. (2006, agosto). "Cultura e Conhecimento Corporativo". Espacios, Vol. 27, No. 2, pág. 35-49.

Yahyagil, M. (2006). "The fit between the concepts of organizational culture and climate", en Journal of Organizational Culture, Communications and Conflict.

Zammuto, R. F \& Krakower, J. Y. (1991), "Quantitative and qualitative studies of organizational culture" en Richard W. Woodman y William A. Pasmore, Research in Organizational Change and Development. Vol. 5. págs 83-114.

Zurbriggen, C. (2006). "El institucionalismo centrado en los actores: una perspectiva analítica en el estudio de las políticas públicas", en Revista de Ciencia Política, Vol. 26, No. 1, págs. 67-83. 
